\title{
X-RAY ABSORPTION NEAR EDGE STRUCTURE ANALYSIS OF HgMnSe, HgFeSe AND HgTeSe*
}

\author{
E. Burattini
}

Laboratori Nazionali di Frascati INFN, Frascati, Italy

A. Kisiel, R. MaRKowski

Instytut Fizyki, Uniwersytet Jagielloński, Kraków, Poland

\author{
G. Dalba
}

Dipartimento di Fisica, Universita di Trento, Povo, Italy

$$
\text { AND W. GiRIaT }
$$

IVIC Centro de Fisica, Caracas, Apartado 21 827, Venezuela

Dedicated to Professor Dr. Julian Auleytner

on the occasion of his 70th birthday

(Received October 27, 1992)

Experimental studies of the Se $K \mathrm{X}$-ray absorption edges have been carried out in $\mathrm{HgTe}_{0.5} \mathrm{Se}_{0.5}, \mathrm{Hg}_{0.8} \mathrm{Mn}_{0.2} \mathrm{Se}$ and $\mathrm{Hg}_{0.9} \mathrm{Fe}_{0.1} \mathrm{Se}$. A comparison is made for $\mathrm{Hg}_{0.5} \mathrm{Mn}_{0.5} \mathrm{Se}$ and $\mathrm{Hg}_{0.5} \mathrm{Fe}_{0.5} \mathrm{Se}$ theoretical results obtained by the self-consistent, semi-relativistic LMTO method within the LD approximation. Small differences of Se $K$ edge XANES have been shown for HgTeSe and $\mathrm{HgSe}$ in contrary to evident differences for $\mathrm{HgMnSe}$ and $\mathrm{HgFeSe}$. This behaviour of the absorption Se $K$ edges is related to significant, hybridized contribution of the $3 d$ and $4 s \mathrm{Mn}$ or Fe unoccupied states. The maximal contributions of the hybridized bands of the hypothetic zinc-blende $\mathrm{MnSe}$ or FeSe were estimated using the virtual crystal model. These maximal contributions are situated at about $6 \mathrm{eV}$ and $17 \mathrm{eV}$ in zinc-blende $\mathrm{MnSe}$ and at $-0.8 \mathrm{eV}$ and $5.5 \mathrm{eV}$ in zinc-blende FeSe. For HgFeSe apart from the evidence of the strongly localized resonant donor (empty for low Fe concentrations) the complementary hybridized contribution of Fe $3 d$ empty states also situated around the conduction band minimum should be taken into consideration. PACS numbers: 78.70.Dm

*This work is in part supported by the programme No. PB 2528/2/91 of the Committee for Scientific Research. 


\section{Introduction}

The aim of this paper is to present the X-ray Absorption Near Edge Structure (XANES) analysis of $\mathrm{HgSe}$ and $\mathrm{HgTeSe}$, as well as $\mathrm{HgFeSe}$ and $\mathrm{HgMnSe}$. The electronic structure of $\mathrm{HgSe}$ [1-4] and $\mathrm{HgTeSe}$ is characterized by the inverted band structure whereas $\mathrm{HgMnSe}$ [5] and $\mathrm{HgFeSe}$ ternary compounds, in an analysed range of $\mathrm{Mn}$ and $\mathrm{Fe}$ content, possess a direct band structure with narrow energy gap. Moreover, $\mathrm{HgMnSe}$ and $\mathrm{HgFeSe}$ ternary compounds, belonging to the diluted magnetic semiconductors (DMS's) group, similarly to other representatives of the DMS's materials, have interesting semiconducting and magnetic properties [5-7]. All studied materials crystallize in the tetrahedrally ordinated zinc-blende (ZB) crystalline structure. A solubility limit of the transition metals $\mathrm{Mn}$ and $\mathrm{Fe}$ in HgSe to which it grows as the crystalline one-phase ternary compound amounts to $38 \%$ [5] and about $15 \%$ [8], respectively. $\mathrm{HgTe}$ and $\mathrm{HgSe}$ crystallize in the zinc-blende structure [9]. They mix mutually without any limits and always create the zinc-blende one-phase structure of $\mathrm{HgTeSe}$. The binary and ternary compounds possessing an inverted band structure or a direct and narrow energy gap have rather a well-defined energy position of the Fermi level, which is commonly placed close to, or at the absolute minimum of the conduction band, i.e. in the case of the studied ZB compounds at $\Gamma$ point of the Brillouin zone. This fact carries one of the most important information for X-ray absorption edge analysis in the semiconductors.

In general, for semiconductors with wider energy gap, the energy position of Fermi level depends significantly on the doping of crystal, but for the narrow energy gap materials the Fermi energy could be closely related to the energy position of the origin of unoccupied projected density of states of the conduction band. The photoemission spectroscopy studies of the valence band structure for $\mathrm{HgMnSe}$ and $\mathrm{HgFeSe}$ have shown a significant influence of the $\mathrm{Mn}$ and $\mathrm{Fe} 3 d$ occupied states on the electronic structure of the $\mathrm{HgSe}$ host crystal $[8,10,11]$. Also unoccupied $3 d$ states of $\mathrm{Mn}$ and Fe affect significantly the conduction band structure of II-VI group compounds. It has been established that $\mathrm{Mn}$ ions modify the conduction band structure of CdMnTe and ZnMnTe [12,13] as well as Fe ions modify also the conduction band structure of CdFeTe [14]. In the case of $\mathrm{HgFeSe}$ alloy it was shown that $\mathrm{Fe}$ creates in the $\mathrm{HgSe}$ conduction band a resonant donor whose ground state coincides with continuum of the conduction band $[15,16]$. This effect shifts up slightly the Fermi level when Fe content increases.

Recently, the theoretical analysis of the conduction band structure and preliminary comparison with the experimental data of the X-ray absorption edge has been carried out [17]. The one-particle, self-consistent, semi-relativistic LMTO method $[18,19]$ was used for the theoretical analysis. The exchange correlation local spin density approximation (LSDA) potential was taken in the form proposed by Vosko et al. [20]. The calculations were carried out for the antiferromagnetic phase in the hypothetical simple cubic lattice. The calculations of the theoretical $\mathrm{X}$-ray absorption in the absorption edge region agreed satisfactorily with experimental datä, particularly for Se $K$ edge [17]. In this work the analysis of the XANES spectra has been extended on the basis of the unpublished electronic 
structure schematic layouts obtained during the earlier LMTO band structure calculations for $\mathrm{HgSe}$ and antiferromagnetic $\mathrm{Hg}_{0.5} \mathrm{Mn}_{0.5} \mathrm{Se}$ and $\mathrm{Hg}_{0.5} \mathrm{Fe}_{0.5} \mathrm{Se}$ [17]. For the analysis of the $\mathrm{Mn}$ and $\mathrm{Fe}$ contribution to the conduction band structure the recently developed virtual crystal model [21, 22] has been used.

\section{Experiments and results}

X-ray absorption experiments have been carried out with the use of synchrotron radiation at the ADONE WIGGLER facility in Frascati [23] utilizing the Si (220) channel-cut crystal monochromator. The originally produced samples were the high purity monocrystalline $\mathrm{HgSe}$ and ternary compounds $\operatorname{Ig}_{1-x} \mathrm{Mn}_{x} \mathrm{Se}$ $(x=0.2), \mathrm{Hg}_{1-x} \mathrm{Fe}_{x} \mathrm{Se}(x=0.1)$ and $\mathrm{HgTe}_{x} \mathrm{Se}_{1-x}(x=0.5)$. To obtain thin specimens of the controlled thickness and homogeneity as required for X-ray absorption measurements the samples were finely powdered and deposited on polyacetate films. X-ray absorption spectroscopy measurements have been carried out for $\mathrm{Se}$ $K$ edge. In the experiment the "thickness effect" was negligible [24].

The resolution of the experimental set-up of the type used by us is limited by a finite width of the rocking curve of the monochromating crystal. The resulting instrumental Gaussian broadening of the natural width of Se $K$ edge spectrum has been estimated to be $4.6 \mathrm{eV}$. The contribution of each edge to the absorption coefficient has been isolated by extrapolating the pre-edge region to higher energies by a Victoreen-like fit and by subtracting the fitted curve from the remaining experimental spectrum. Figure 1 presents Se $K$ absorption X-ray edges for $\mathrm{HgSe}$ and ternary $\mathrm{HgMnSe}(20 \%$ of Mn), $\mathrm{HgFeSe}(10 \%$ of Fe) and IIgTeSe (50\% of Te).

\section{Discussion}

In the zinc-blende crystalline structure which has tetrahedral coordination, every anion is surrounded by four cations and vice versa, but the cations or anions form second nearest neighbours with themselves. As it has been reported [25] in DMS for II-VI group ternary compounds, the transition metals always substitute cations. Therefore, in our analysis we should expect a stronger interaction between $\mathrm{Se}$ and $\mathrm{Mn}$ or Fe atoms than Se-Se and $\mathrm{Hg}-\mathrm{Mn}(\mathrm{Fe}$ ) or Se-Te (in case of $\mathrm{Ig} \mathrm{TeSe}$ ) because in the crystal the overlap of the wave function between cation and anion is larger than that between anion and anion or cation and cation.

A relatively smaller contribution of the second neighbours interaction was discussed earlier for $\mathrm{ZnMnTe}$ and CdMnTe [14]. This fact imposes a careful analysis of XANES data for Se $K$ edge, i.e. an analysis of the hybridization changes around Se atoms when the Mn or Fe content increases. In Fig. 1 Se $K$ absorption edges for $\mathrm{HgSe}$ (curve $a$ ) and HgTeSe (curve $d$ ) show a general tendency that even large $50 \%$ substitution of $\mathrm{Se}$ by $\mathrm{Te}$ atoms in the $\mathrm{Se}$ anion sublattice in $\mathrm{HgSe}$ causes a very weak modification of the XANES spectrum. It seems clear if we take into consideration that in the crystalline tetrahedral structure Te and Se are always second neighbours and also that the schematic layout for IIgSe presented in Fig. 2 is very similar to the respective layout for HgTe (see Fig. 8 in Ref. [26]). These figures show the schematic layouts of the self-consistent bands calculated by 


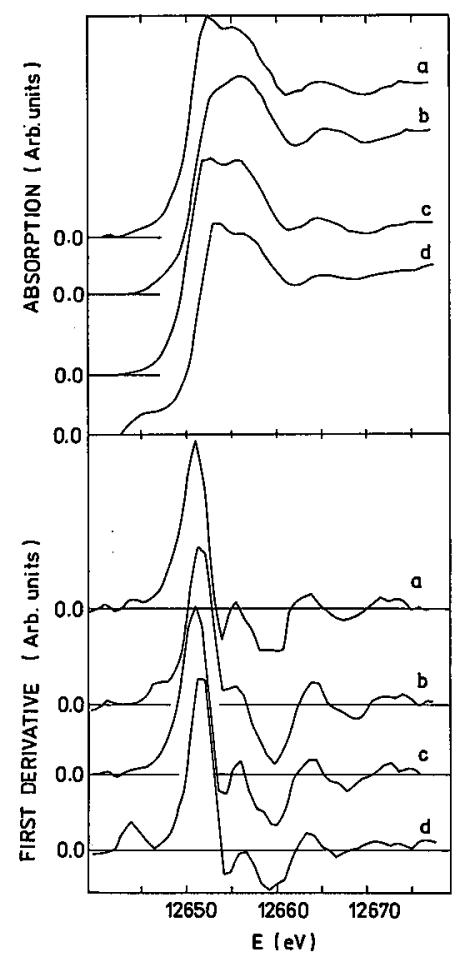

Fig. 1. Experimental X-ray absorption Se $K$ edges and their first derivatives for $\mathrm{HgSe}$ (curve a), $\mathrm{Hg}_{0.8} \mathrm{Mn}_{0.2} \mathrm{Se}$ (curve b), $\mathrm{Hg}_{0.09} \mathrm{Fe}_{0.1} \mathrm{Se}$ (curve $c$ ) and $\mathrm{HgTe}_{0.5} \mathrm{Se}_{0.5}$ (curve d).

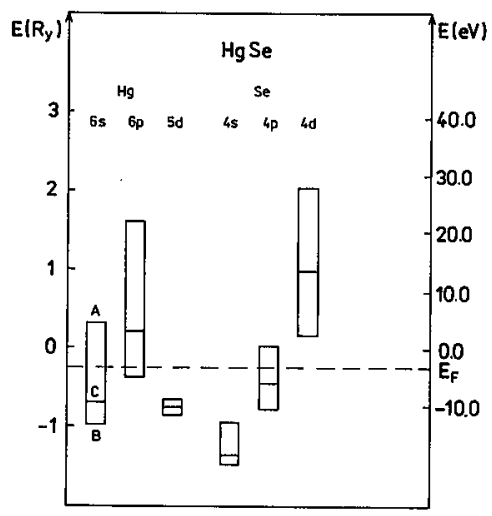

Fig. 2. Schematic layout of the electronic structures of HgSe. The bars depict the extend of the $n, l$ bands as defined by the parameters $A$ (top of the band), $C$ (band centre) and $B$ (bottom of the band). 
LMTO method and are deduced from the potential-parameter-related quantities $B_{l}, C_{l}$ and $A_{l}$ that specify the bottom (bonding states), mass centre and top (antibonding states) of the $l$ band, respectively. In the schematic layouts for $\mathrm{HgSe}$ and $\mathrm{HgTe}$, the most significant contribution of the Se $4 d$ and $\mathrm{Te} 5 d$ bands are extended in the similar energy range and the centre of the band $\left(C_{l}\right)$ is placed for both compounds in almost the same energy position. In contrary to this, the curves $b$ and $c$ in Fig. 1 show that the Se $K$ edge XANES spectra for HgMnSe and $\mathrm{HgFeSe}$ ternary compounds, even for relatively lower content of $\mathrm{Mn}(20 \%)$ or $\mathrm{Fe}(10 \%)$, differ evidently. Se $K$ edge is the result of a transition from $1 s$ core state to $p$-like projected density of states (DOS) of the conduction band. Therefore, the significant differences in the shape and intensities of the XANES spectra is the evident result of the $s-p-d$ hybridization in the conduction band. Particularly, the conduction band DOS is modified by the presence of $\mathrm{Mn}$ or Fe $3 d$ empty states. These differences are related certainly to substitution of $\mathrm{Hg}$ by $\mathrm{Mn}$ or $\mathrm{Fe}$ ions, i.e. the substitution of $\mathrm{Hg} 6 s$ and $6 p$ bands by $4 s, 4 p$ and partially occupied $3 d$ states of $\mathrm{Mn}$ or $\mathrm{Fe}$.
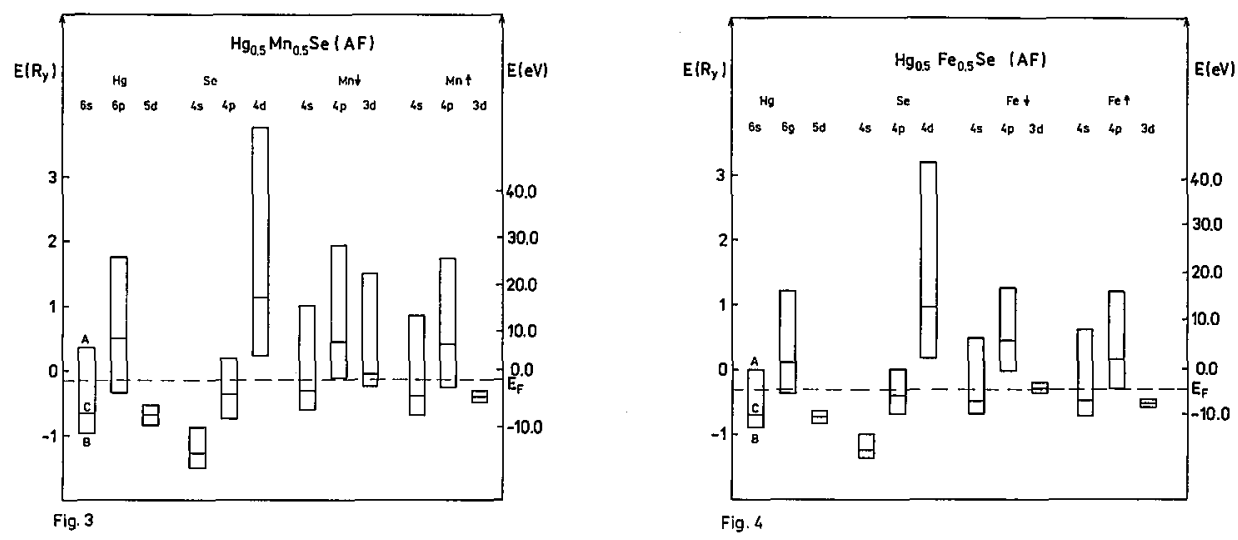

Fig. 3. Schematic layout of the electronic structure of $\mathrm{Hg}_{0.5} \mathrm{Mn}_{0.5} \mathrm{Se}$. The bars depict the extend of the $n, l$ bands as defined by the parameters $A$ (top of the band), $C$ (band centre) and $B$ (bottom of the band).

Fig. 4. Schematic layout of the electronic structure of $\mathrm{Hg}_{0.5} \mathrm{Fe}_{0.5} \mathrm{Se}$. The bars depict the extend of the $n, l$ bands as defined by the parameters $A$ (top of the band), $C$ (centre of the band) and $B$ (bottom of the band).

A comparison of the schematic layouts for $\mathrm{HgMnSe}$ and $\mathrm{HgFeSe}$ presented in Figs. 3 and 4 with schematic layout for HgSe (Fig. 2) confirms a considerable contribution of the transition metals in XANES spectra of the diluted magnetic semiconductors. To extract a contribution which comes from the hybridized electronic states of $\mathrm{Mn}^{2+}$ or $\mathrm{Fe}^{2+}$ ions in ternary compounds we used a virtual-crystal model checked earlier for other II-VI compounds [13, 22]. 
If a simple formula

$\mathrm{A}_{1-x}^{\mathrm{II}} \mathrm{B}_{x} \mathrm{C}^{\mathrm{VI}}-(1-x) \mathrm{A}^{\mathrm{II}} \mathrm{C}^{\mathrm{VI}}=x \mathrm{BC}^{\mathrm{VI}}$

is applied to $\mathrm{HgMnSe}$ and $\mathrm{HgFeSe}$, where $\mathrm{A}$ and $\mathrm{C}$ are $\mathrm{Hg}$ and Se atoms, respectively, and B stands for $\mathrm{Mn}$ or Fe atoms, one can obtain the experimental Se $K$ edge XANES for a hypothetical zinc-blende $\mathrm{MnSe}$ and FeSe structure.

In case of CdMnTe the definition and analysis of the basic properties of the zinc-blende hypothetical MnTe has been carried out by Wei and Zunger [21] and then extended in several our papers [13, 14]. A hypothetical ZB MnTe extracted from experimental data for $\mathrm{ZnMnTe}$ and $\mathrm{CdMnTe}$ agreed excellently with anticipated theoretical calculations [14].
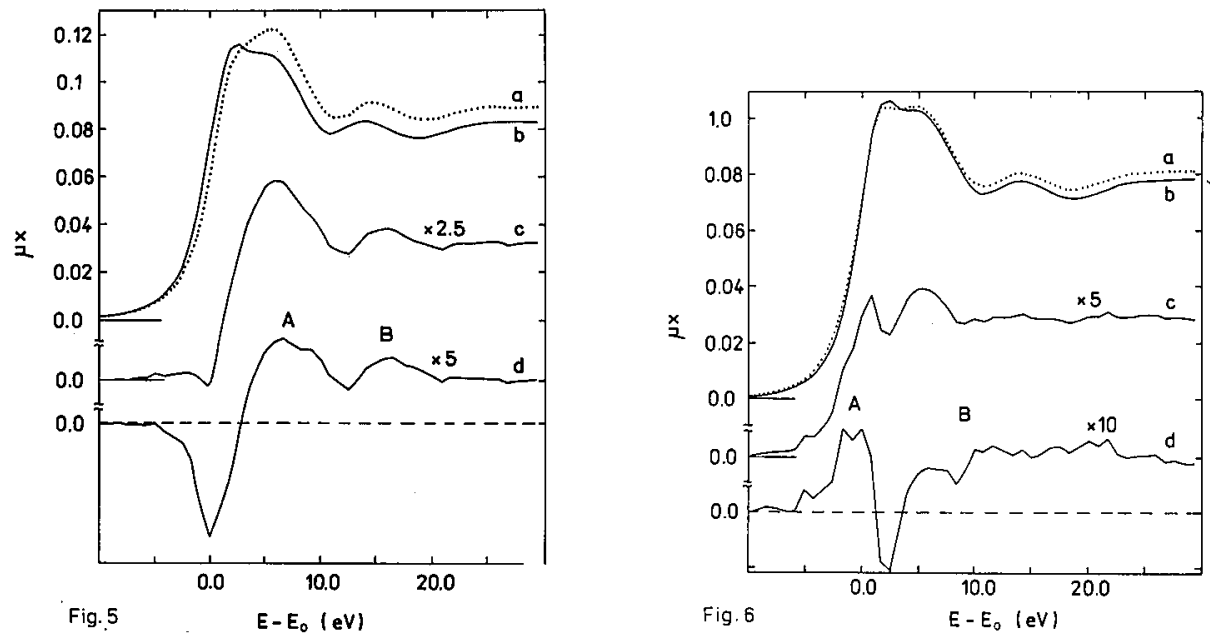

Fig. 5. Virtual crystal model analysis of the experimental X-ray absorption Se $K$ edges for HgMnSe. Curve a (dotted line) - absorption coefficient for HgMnSe in reduced energy scale, curve $b$ - for comparison $\mathrm{HgSe}$, curve $c$ - absorption edge of the hypothetical zinc-blende MnSe extracted with the use of the virtual crystal model, curve $d-$ a contribution of the hybridized Mn $3 d$ and $4 s$ electronic states in $\mathrm{HgMnSe}$.

Fig. 6. Virtual crystal model analysis of the experimental X-ray absorption edge for $\mathrm{HgFeSe}$. Curve a (dotted line) - absorption coefficient for $\mathrm{HgFeSe}$ in reduced energy scale, curve $b-$ for comparison $\mathrm{HgSe}$, curve $c$ - absorption edge of the hypothetic zinc-blende FeSe extracted with the use of the virtual crystal model, curve $d-$ a contribution of the hybridized $\mathrm{Fe} 3 d$ and $4 s$ electronic states in $\mathrm{HgFeSe}$.

Figures 5 and 6 show Se $K$ edges for hypothetical ZB MnSe and FeSe compounds (curves $c$ ). The curves $d$ on these figures present a maximum contribution of the $\mathrm{MnSe}$ and $\mathrm{FeSe}$ in $\mathrm{HgMnSe}$ and $\mathrm{HgFeSe}$, respectively. In case of $\mathrm{ZB} \mathrm{MnSe}$ a maximum $p$-like contribution is localized at about $6 \mathrm{eV}$ (maximum $A$ ) and $17 \mathrm{eV}$ (maximum $B$ ) above $\mathrm{HgSe}$ inflection point $E_{0}$. The schematic layout of electron structure of unoccupied states of $\mathrm{Hg}_{0.5} \mathrm{Mn}_{0.5} \mathrm{Se}$ (Fig. 3) shows that the greatest contribution to the maximum $A$ comes from hybridized $4 p, 4 s$ and $3 d$ states of $\mathrm{Mn}$ 
and some contribution from Se $4 d$ states, the maximum $B(17 \mathrm{eV})$ comes mainly from Se $4 d$ states, Mn $4 p$ and in small part from Mn $3 d$. This comparison seems to be acceptable even through the schematic layout that has been calculated for $50 \% \mathrm{Mn}$ content, because the limits of the $n, l$ bands should change slightly with the transition metal content.

The ZB FeSe $p$-like contribution possesses maxima localized at about $-0.8 \mathrm{eV}$ and $5.5 \mathrm{eV}$ under and above the inflection point $E_{0}$ for $\mathrm{HgSe}$, respectively. A comparison of the FeSe contribution in $\mathrm{HgFeSe}$ with a respective schematic layout of the electronic structure calculated for $\mathrm{Hg}_{0.5} \mathrm{Fe}_{0.5} \mathrm{Se}$ shows that a maximum situated closely to $E_{0}$ should be related to $\mathrm{Fe} 4 s$ and the strongly localized $\mathrm{Fe} 3 d$ empty states as well as some contribution of the $\mathrm{Se} 4 p$ antibonding states. It means that the significant contribution of the $\mathrm{Fe} 3 d$ unoccupied states, which hybridize with $s$ and $p$-like DOS appears at the gap or closely to the absolute minimum of the conduction band and should be discussed complementarily to the reported earlier localized Fe state named as a resonant donor $[15,16]$.

\section{Conclusions}

A XANES analysis of the HgTeSe, HgMnSe and HgFeSe has shown that for $\mathrm{HgSe}$ and $\mathrm{HgTe}_{0.5} \mathrm{Se}_{0.5}$ the absorption curves do not change significantly, but for ternary compounds with Mn or Fe the XANES differs much in comparison to binary $\mathrm{HgSe}$. This behaviour of the absorption edges has been related to the significant contribution of the $3 d \mathrm{Mn}$ or Fe unoccupied states. The maximal contributions of the hybridized bands of hypothetic zinc-blende MnSe or FeSe were estimated using the virtual crystal model. These maximal contributions are situated at about $6 \mathrm{eV}$ and $17 \mathrm{eV}$ in $\mathrm{ZB}$ MnSe and at $-0.8 \mathrm{eV}$ and $5.5 \mathrm{eV}$ in $\mathrm{ZB}$ $\mathrm{FeSe}$. For $\mathrm{HgFeSe}$ apart from the evidence of the strongly localized resonant donor (empty for low $\mathrm{Fe}$ concentrations) $[15,16]$ the complementary hybridized contribution of Fe $3 d$ empty states also situated around the conduction band minimum should be taken into consideration.

\section{Acknowledgments}

We would like to thank Dr. A. Balerna and the technical staff of the Project Wiggler ADONE (PWA) Group of the Laboratori Nazionali di Frascati for their help and hospitality. One of us (A.K.) gratefully acknowledges the National Institute of Nuclear Physics (INFN) in Frascati for financal support during the initial preparatory stage of this work.

\section{References}

[1] J.G. Broerman, Phys. Rev. 183, 754 (1969).

[2] D.G. Seiler, R.R. Gałazka, W.M. Becker, Phys. Rev. B 3, 4274 (1971).

[3] S.L. Lehoczky, J.G. Broerman, D.A. Nelson, C.R. Whitsett, Phys. Rev. B 9, 1958 (1974). 
[4] A. Mycielski, J. Kossut, M. Dobrowolska, W. Dobrowolski, J. Phys. C, Solid State Phys. 15, 3293 (1982).

[5] J.K. Furdyna, J. Vac. Sci. Technol. A 4, 2002 (1986).

[6] A. Lewicki, J. Spałek, A. Mycielski, J. Phys. C, Solid State Phys. 20, 2005 (1987).

[7] H.J.M. Swagten, F.A. Arnouts, A. Twardowski, W.J.M. de Jonge, A. Mycielski, Solid State Commun. 69, 1047 (1989).

[8] A. Mycielski, P. Dzwonkowski, B. Kowalski, B.A. Orłowski, M. Dobrowolska, M. Arciszewska, W. Dobrowolski, J.M. Baranowski, J. Phys. C, Solid State Phys. 19, 3605 (1986).

[9] N.Kh. Abrikosov, V.F. Bankina, L.V. Poretskaya, L.E. Shelimova, E.V. Skudova, Semiconducting II-VI, IV-VI and V-VI Compounds, Plenum Press, New York 1969.

[10] B. Kowalski, V. Chab, B.A. Orłowski, J. Majewski, A. Sarem, A. Mycielski, 'Acta Phys. Pol. A 73, 455 (1988).

[11] A. Franciosi, S. Chang, C. Caprile, R. Reifenberger, U. Dębska, J. Vac. Sci. Technol. A 3, 926 (1985).

[12] A. Kisiel, M. Piacentini, F. Antonangeli, J. Oleszkiewicz, A. Rodzik, N. Zema, A. Mycielski, J. Phys. C, Solid State Phys. 20, 5601 (1987).

[13] A. Kisiel, A.-I. Ali Dahr, P.M. Lee, G. Dalba, P. Fornasini, E. Burattini, Phys. Rev. B 44, 11075 (1991-II).

[14] A. Kisiel, P.M. Lee, E. Burattini, G. Dalba, P. Fornasini, W. Giriat, Solid State Commun. 81, 151 (1992).

[15] M. Arciszewska, A. Mycielski, M. Dobrowolska, W. Dobrowolski, J.M. Baranowski, W. Warmiński, B. Witkowska, U. Blinowska, Acta Phys. Pol. A 69, 989 (1986).

[16] R. Reifenberger, J. Kossut, J. Vac. Sci. Technol. A 5, 2987 (1987).

[17] A. Kisiel, J. Oleszkieiwcz, J. Goniakowski, R. Markowski, E. Burattini, G. Dalba, F. Rocca, Acta Phys. Pol. A 80, 373 (1991).

[18] O.K. Andersen, Phys. Rev. B 12, 3060 (1975).

[19] H.L. Skriver, The LMTO Method, Solid State Sciences, Vol. 41, Springer Verlag, Berlin 1984.

[20] S.H. Vosko, L. Wilk, M. Nusair, Can. J. Phys. 58, 1200 (1980).

[21] Su-Huai Wei, A. Zunger, Phys. Rev. Lett. 56, 2391 (1986).

[22] A. Kisiel, A.-I. Ali Dahr, P.M. Lee, G. Dalba, P. Fornasini, E. Burattini, Phys. Rev. B 42, 11114 (1990-I).

[23] E. Burattini, E. Bernieri, A. Balerna, C. Mencuccini, R. Rinzivillo, G. Dalba, P. Fornasini, Nucl. Instrum. Phys. Res. A 246, 125 (1986).

[24] E.A. Stern, K. Kim, Phys. Rev. B 23, 3781 (1981); L.G. Parratt, C.F. Hempstead, E.L. Jossem, Phys. Rev. 105, 1228 (1957).

[25] A. Balzarotti, M.T. Czyżyk, A. Kisiel, N. Motta, M. Podgórny, M. Zimnal-Starnawska, Phys. Rev. B 30, 2295 (1984); ibid., 31, 7526 (1985).

[26] A. Kisiel, G. Dalba, P. Fornasini, M. Podgórny, J. Oleszkiewicz, F. Rocca, E. Burattini, Phys. Rev. B 39, 7895 (1989-I). 